\title{
Interference Control Mechanism for 5G Indoor Micro Operators Utilizing Dynamic TDD
}

\author{
Kimmo Hiltunen and Marja Matinmikko-Blue \\ Centre for Wireless Communications \\ University of Oulu \\ Oulu, Finland \\ kimmo.hiltunen@oulu.fi; marja.matinmikko@oulu.fi
}

\begin{abstract}
Future 5G networks will increasingly target local small cell deployments complementing the coverage of the existing outdoor networks. To make the high-quality inbuilding deployments more cost-efficient, and to promote innovation and competition in the market, new local business and spectrum authorization models are needed. Recently proposed micro operator concept allows the establishment of building-specific $5 \mathrm{G}$ networks by different stakeholders through the availability of local spectrum micro licensing. However, in order to make the concept of local high-quality $5 \mathrm{G}$ networks feasible, impact of the interoperator interference on the performance of the victim micro operator needs to be understood. This paper presents system simulation results demonstrating how the required minimum separation distance between two uncoordinated micro operators utilizing dynamic TDD in the $3.6 \mathrm{GHz}$ band can be decreased by controlling the base station transmission power of the interfering network based on the measurements performed by the victim network. Without any interference control mechanism the required minimum separation distance can become as large as $\mathbf{4 0 0}$ meters, but with the proposed mechanism the required separation distance can be more than halved, or even made equal to zero. It is also shown that the interference threshold resulting in the smallest minimum separation distance is highly scenario-dependent: it will depend both on the network layout and load of both the victim and the interfering micro operator.
\end{abstract}

Index Terms - 5G, micro operator, indoor, dynamic TDD, interference control, spectrum sharing.

\section{INTRODUCTION}

Mobile communication networks have been traditionally deployed by mobile network operators (MNO) with exclusive spectrum licenses to provide wide area coverage. Future $5 \mathrm{G}$ networks will increasingly target local in-building systems, which changes the traditional mobile communication market roles [1]. Locally deployed 5G networks will play an increasing role in the provisioning of new traffic-heavy services in vertical sectors' use cases, and for deployments in the higher frequency bands. Instead of relying solely on the networks deployed by the MNOs, an emerging solution gaining increasing attention in research, regulation and industry is to allow other stakeholders, for example the venue owners, to deploy and operate their own inbuilding networks to satisfy local capacity and coverage needs [1]. These local high-quality wireless networks aim at providing guaranteed quality of service addressing vertical sectors' service requirements in confined areas [2]. It is further envisioned that the deployment of these local high-quality networks will take place on shared spectrum bands [3].

A micro operator concept [4] has recently been introduced to allow different stakeholders to deploy local high-quality $5 \mathrm{G}$ networks. This requires that the availability of local spectrum for reliable service delivery is secured with the help of some form of micro licensing model [5][6] and the regulators are taking steps towards local 5G spectrum licenses. Micro operators aim at serving their own restricted customer set and at the same time they can act as neutral hosts for the customers of the overlying MNOs [7]. The introduction of a potentially large number of local indoor micro operators requires some form of interference control between the local license holders and the potential incumbent spectrum users to guarantee that their operations remain free from harmful interference. In particular, this will be the case if the aim is to allow the micro operators to fully benefit from the flexibility offered by the dynamic time division duplex (TDD), which is one of the key features of the $5 \mathrm{G}$ New Radio [8].

An initial analysis of the interference between adjacent micro operator deployments in the $3.6 \mathrm{GHz}$ band was presented in [5] where the separation distance between two micro operators in adjacent buildings was analyzed. The work was continued in [9], and further in [10], where the impact of inter-operator interference on the micro operator network performance was evaluated. Among other things, the results in [10] demonstrated that the interference received from the other micro operator's base stations was the main cause for both the downlink and uplink performance losses.

This paper continues the work in [10] by introducing a simple interference control mechanism to reduce the impact of the inter-operator interference on both the downlink and the uplink performance of the victim micro operator. The goal is to evaluate the impact of the 


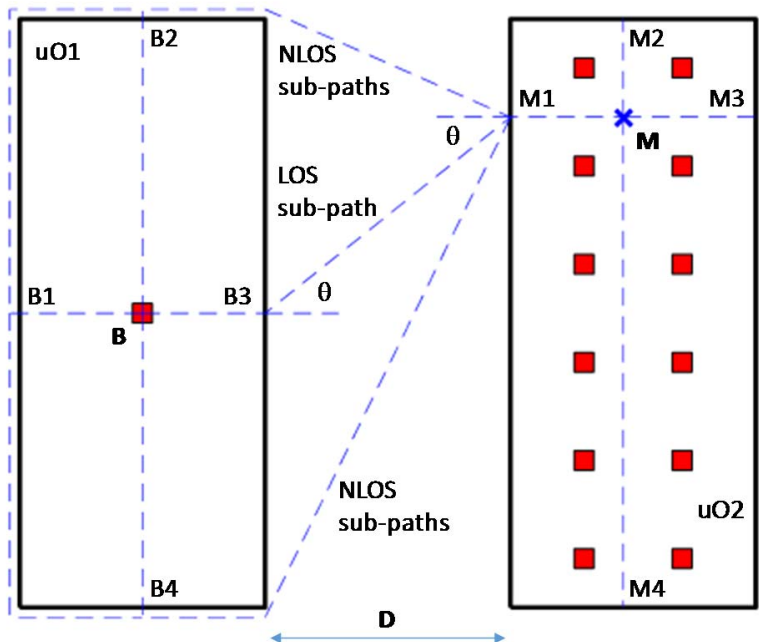

Fig. 1. Assumed network layout and the model for building-tobuilding propagation.

interference control mechanism on the required minimum separation distance between two uncoordinated indoor micro operators utilizing dynamic TDD and sharing the same channel in the $3.6 \mathrm{GHz}$ band.

The rest of this paper is organized as follows. Section II introduces the system model, including the network layout, inter-operator interference scenarios, propagation models, and models for the radio resource management and the average user performance. Then, system simulation results evaluating the performance of the victim micro operator and the required minimum separation distance are presented and analyzed in Section III. Finally, some conclusions are drawn and a few future research topics are discussed in Section IV.

\section{SYSTEM MODEL}

Our system model considers the deployment of local $5 \mathrm{G}$ indoor micro operator networks that are sharing the same spectrum band. In the following, a brief description of the assumed network layout is given, followed by a brief discussion of the different inter-operator interference scenarios for the deployment of high-quality wireless networks by two neighboring micro operators. To evaluate the corresponding interference levels, the applied propagation models, both for the indoor propagation within a building and for the building-tobuilding propagation between the different micro operators, are also presented. Finally in order to characterize the impact of the interference, models for radio resource management and average user performance are discussed. A more detailed discussion of the assumed deployment scenario and the propagation modeling can be found in [11].

\section{A. Network Layout}

The micro operator network deployment is assumed to consist of two equally-sized buildings $(50 \times 120 \mathrm{~m}$, based on the indoor deployment model defined in [12]),

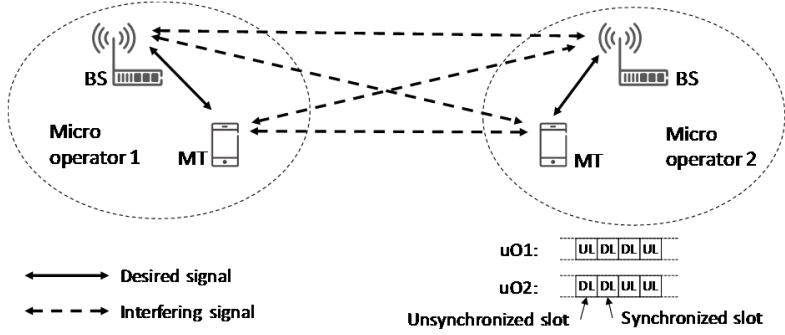

Fig. 2. Different inter-operator interference scenarios between neighboring micro operators [9][10].

located at a distance $D$ from each other as depicted in Fig. 1. The buildings are assumed to be in line-of-sight (LOS) with each other and only one floor per building is modeled. Micro operator $1(\mathrm{uO} 1)$ is assumed to be serving users within the first building, while micro operator $2(\mathrm{uO} 2)$ is serving users within the second building. Furthermore, $\mathrm{uO} 2$ has deployed 12 base stations per floor (with the same network layout as in [12]), while the density of base stations belonging to $\mathrm{uO} 1$ is varied during the evaluations. Finally, both indoor networks are assumed to be operating on the same channel in the 3.6 $\mathrm{GHz}$ band.

\section{B. Inter-Operator Interference Scenarios}

Local 5G micro operator deployments face various interference scenarios depending on the spectrum where they operate and the operational characteristics of the networks. For example, the applied duplexing method, traffic charateristics and the level of coordination between the co-existing networks have a major impact on the characteristics of the inter-operator interference.

The following four different inter-operator interference scenarios between local $5 \mathrm{G}$ micro operators can be identified, as depicted in Fig. 2 [9][10]:

- Interference from base station (BS) to mobile terminal (MT) (downlink-to-downlink (DL-toDL) interference), valid for both coordinated and uncoordinated TDD as well as for frequency division duplex (FDD).

- Interference from MT to BS (uplink-to-uplink (UL-to-UL) interference), valid for both coordinated and uncoordinated TDD as well as for FDD.

- Interference from BS to BS (DL-to-UL interference), valid for uncoordinated TDD.

- Interference from MT to MT (UL-to-DL interference), valid for uncoordinated TDD.

The system model assumed in this paper will consider all four interference scenarios when evaluating the impact of the inter-operator interference on the performance of the victim micro operator. It is further assumed that the cells within an operator's network are coordinated (or synchronized), while the cells belonging to different operators are uncoordinated (or unsynchronized). 


$$
\begin{aligned}
& \gamma_{m, b, r}=\frac{P_{b, r} C_{m, b}^{-1}}{I_{o w n, m, b, r}+I_{o t h e r, m, r}+I_{m t 2 m t, m, r}+N_{m, r}}=\frac{P_{b, r} C_{m, b}^{-1}}{\sum_{\substack{j=1 \\
j \neq b}}^{B} \frac{\tau_{j, r} P_{j, r}}{C_{m, j}}+\sum_{k=1}^{K} \frac{\tau_{k, r} P_{k, r}}{C_{m, k}}+\sum_{s=1}^{S} \frac{\tau_{s, r} P_{M, s, r}}{C_{m, s}}+N_{m, r}} \\
& \gamma_{m, b, r}=\frac{P_{M, m, b, r} C_{m, b}^{-1}}{I_{o w n, m, b, r}+I_{o t h e r, b, r}+I_{b s 2 b s, b, r}+N_{b, r}}=\frac{P_{M, m, b, r} C_{m, b}^{-1}}{\sum_{\substack{t=1 \\
t \neq m}}^{T} \frac{\tau_{t, r} P_{M, t, r}}{C_{t, b}}+\sum_{s=1}^{S} \frac{\tau_{s, r} P_{M, s, r}}{C_{s, b}}+\sum_{k=1}^{K} \frac{\tau_{k, r} P_{k, r}}{C_{k, b}}+N_{b, r}}
\end{aligned}
$$

\section{Propagation Models}

Interference evaluations require proper modeling of propagation between the two neighboring micro operators, where the coupling loss $C_{m, b}$ between a mobile terminal $m$ and a base station $b$ can be calculated as

$$
C_{m, b, d B}=L_{m, b}-G_{B S, m, b}-G_{M T, m, b}+X_{m, b}
$$

In (1), $G_{B S, m, b}$ and $G_{M T, m, b}$ are the base station and mobile terminal antenna gains, respectively, and $X_{m, b}$ is a log-normally distributed random variable modeling the impact of shadow fading. Next, the assumed modeling of the path loss $L_{m, b}$ for our $5 \mathrm{G}$ indoor micro operator deployment is discussed in more detail.

The indoor propagation within a building is modeled using the 3GPP Indoor - Mixed Office propagation model as defined in [12]. The path loss model includes both a LOS and a non-line-of-sight (NLOS) component, and the LOS probability is defined to decrease as a function of the distance between the base station and the mobile terminal. The standard deviation of the shadow fading is assumed to be equal to $3 \mathrm{~dB}$ (LOS) or $8 \mathrm{~dB}$ (NLOS) [12]. Furthermore, both the shadow fading and the LOS probability are assumed to be spatially correlated, with correlation distances equal to $10 \mathrm{~m}$ or 6 $\mathrm{m}$ (shadow fading in LOS or NLOS), and $10 \mathrm{~m}$ (LOS probability) [12].

The model for the path losses between the different buildings assumes four different sub-paths for each building, one through each building wall [13], as shown in Fig. 1. The total received power for each link between a transmitter and a receiver is calculated as a linear sum of the received powers from all resulting 16 different subpaths. Each sub-path takes into account both the outdoor path loss between the outer wall reference points (B1-B4 and M1-M4 in Fig. 1), and the building penetration and indoor losses for both buildings. Thus, the path loss per sub-path is calculated as

$$
\begin{aligned}
L\left(f_{c}\right)_{d B}=L_{i n, 1} & +L_{o w, 1}\left(f_{c}\right)+L_{o u t}\left(f_{c}\right)+L_{o w, 2}\left(f_{c}\right) \\
& +L_{i n, 2}
\end{aligned}
$$

In (2), $L_{\text {in }}$ is the indoor loss, modeled as $L_{\text {in }}=$ $0.5 d_{2 D-i n}$, where $d_{2 D-i n}$ is the two-dimensional distance between the indoor node and the outer wall reference point in meters [12]. Parameter $L_{\text {ow }}$ is used to characterize the building wall loss and it consists of two parts: one that depends on the angle of incidence $\theta$ and the other that depends on the wall material and the center frequency $f_{c}$. Depending on the desired building penetration model, $L_{o w}$ is calculated either as described in (3) [14] or in (4) [12].

$$
\begin{gathered}
L_{\text {ow }, \text { LOS }}=20 \cdot(1-\cos \theta)^{2}+L_{\text {material }}\left(f_{c}\right) \\
L_{\text {ow }, N L O S}=5+L_{\text {material }}\left(f_{c}\right)
\end{gathered}
$$

The building wall loss $L_{o w}$ is based on (3) for outer wall reference points B3 and M1, while it is based on (4) for all the other outer wall reference points as illustrated in Fig. 1. The model for the loss due to the wall material $L_{\text {material }}$ is taken from [12], and the selected value equal to $7.7 \mathrm{~dB}$ assumes that the penetrated building walls consist in average of 30\% multi-pane windows and 70\% concrete.

Parameter $L_{\text {out }}$ in (2) is the outdoor path loss between the outer wall reference points. In case of the LOS subpath between B3 and M1 shown in Fig. 1, $L_{\text {out }}$ is based on a free space propagation model, and the applied distance is the sum of the outdoor and indoor distances [13][15]. In case of NLOS sub-paths, $L_{\text {out }}$ considers only the path between the outer wall reference points, and the path loss is based on the recursive microcell model [16], with a breakpoint for the path loss exponent at $300 \mathrm{~m}$. Finally, the shadow fading model assumes that the standard deviation is equal to $6 \mathrm{~dB}$ and the correlation distance is equal to $10 \mathrm{~m}$.

\section{Radio Resource Management and User Performance}

Next, we proceed to model the impact of the interference on the performance of the micro operator networks. In our system model, a user is assumed to be connected to the closest base station belonging to the serving micro operator. Furthermore, a round-robin scheduler is assumed, either in time (DL) or in frequency domain (UL).

The downlink signal-to-interference-and-noise-ratio (SINR) for mobile terminal $m$, being served by base station $b$ on resource block (RB) $r$, is calculated as shown in (5). There, $I_{\text {own }}$ characterizes the intra-operator interference including the received power from all the other base stations belonging to the serving micro operator (having a total of $B$ base stations). $I_{\text {other }}$ is the received power from all base stations and $I_{m t 2 m t}$ is the received power from all mobile terminals belonging to the other micro operators characterizing the inter- 
operator interference. In case of the DL-to-DL interference scenario, $I_{\text {other }} \geq 0$ and $I_{m t 2 m t}=0$, while for the UL-to-DL interference scenario the situation is the opposite: $I_{\text {other }}=0$ and $I_{m t 2 m t} \geq 0$. In more detail, parameter $P_{b, r}$ is the transmission power from base station $b$ on $\mathrm{RB} r, C_{m, b}$ is the coupling loss between mobile terminal $m$ and base station $b, N_{m, r}$ is the thermal noise power per RB of mobile terminal $m$ and $\tau_{j, r}$ is an activity factor indicating whether transmitter $j$ is occupying $\mathrm{RB} r: \tau_{j, r}=1$ if $j$ is transmitting on $r$, otherwise $\tau_{j, r}=0$.

The total transmission power $P_{M, m, b}$ for mobile terminal $m$, served by base station $b$ and occupying a total of $M_{m, b}$ RBs is calculated as

$$
\begin{aligned}
& P_{M, m, b, d B m}=P_{0, b}+10 \log _{10}\left(M_{m, b}\right)+\alpha_{b} C_{m, b, d B}, \\
& P_{M \min } \leq P_{M, m, b} \leq P_{M \max }
\end{aligned}
$$

where $P_{0}$ is the target for the received uplink power per $\mathrm{RB}$ and $\alpha$ is the path loss compensation factor. Finally, uplink SINR is calculated based on (7), where $I_{b s 2 b s}$ is the total received power from base stations belonging to the other micro operators. In case of the UL-to-UL interference scenario, $I_{\text {other }} \geq 0$, while $I_{b s 2 b s}=0$. However, for the DL-to-UL interference scenario, $I_{\text {other }}=0$ and $I_{b s 2 b s} \geq 0$.

After the SINR values have been defined for the occupied RBs, the average SINR value $\left(\bar{\gamma}_{m, b}\right)$ for the active link is calculated as a logarithmic average of the $\gamma_{m, b, r}$ values. Finally, the average SINR is mapped to a corresponding average user throughput as

$R_{m, b}=\frac{0.8 \cdot \mu \cdot \beta_{m, b} \cdot \min \left(R_{\max }, \log _{2}\left(1+\frac{\bar{\gamma}_{m, b}}{1.6}\right)\right)}{u_{b}}$

The throughput model in (8) assumes that the average overhead due to control channels and data retransmissions is equal to $20 \%$ and the margin due to receiver imperfections is equal to $2 \mathrm{~dB}$. Parameter $\mu$ indicates the average usage of the downlink and the uplink as a function of time. The maximum spectral efficiency $R_{\max }$ is defined by the highest available modulation and coding rate, as well as the maximum number of parallel data streams for each link. The impact of scheduling on the average user throughput is taken into account via parameters $\beta_{m, b}$ and $u_{b}$ : for downlink with time domain scheduling $\beta_{m, b}$ is equal to the channel bandwidth (total number of RBs $\times$ bandwidth of a RB), while $u_{b}$ is equal to the total number of users served by base station $b$. For uplink with frequency domain scheduling, $\beta_{m, b}$ is the bandwidth allocated to user $m$, while $u_{b}$ is equal to one.

\section{E. Inter-Operator Interference Control}

A simple model for the inter-operator interference control is assumed during the simulations. In the model, each base station $(b=1, \ldots, B)$ belonging to micro operator A estimates the total received power from all the base stations $(k=1, \ldots, K)$ belonging to micro operator $\mathrm{B}$. The maximum of these measured values is then compared to a predefined interference threshold $I_{t h}$ to define the transmission power scaling factor for micro operator B. Hence, for all values of $k$

$$
\begin{aligned}
& P_{k, d B m}=I_{t h, d B m}-10 \log _{10}\left(\max _{b \in B}\left(\sum_{k=1}^{K} \frac{\tau_{k}}{C_{k, b}}\right)\right), \\
& P_{B \text { Smin }} \leq P_{k} \leq P_{B \text { Smax }}
\end{aligned}
$$

As a default, the inter-operator interference control is based on the estimated worst case interference, assuming that the interfering network is fully loaded $\left(\tau_{k}=1\right.$ for all values of $k$ ). However, performance of an alternative model where the interference control is based on the actual interference $\left(\tau_{k}=1\right.$ if base station $k$ is active, otherwise $\left.\tau_{k}=0\right)$ is also briefly evaluated.

\section{EVALUATION RESULTS}

This section presents system simulation results characterizing the impact of inter-operator interference between two micro operators, and assuming the use of the simple interference control mechanism as described above. The main simulation parameters have been listed in Table I. For all the system simulations in this paper, we assume that $\mathrm{uO} 1$ is the victim operator, while $\mathrm{uO} 2$ is the interfering operator. Furthermore, the $\mathrm{uO} 2$ network is assumed to consist of 12 base stations, and the number of active users is set so that the average network load is equal to $90 \%$. Finally, a random allocation of DL and UL slots is assumed for both networks, resulting in a 50:50 $\mathrm{UL}: \mathrm{DL}$ ratio in average.

The impact of inter-operator interference is measured by comparing the average user throughput for the multioperator scenario to the corresponding value for the single operator scenario. Furthermore, the impact is measured also by evaluating the required minimum separation distance (MSD) between the different micro operators (or more specifically, between the different buildings) so that the observed average user performance within the victim operator's network is not degraded more than the desired threshold of $1 \%, 2 \%$ or $5 \%$.

The assumed interference control mechanism limits the worst case downlink interference received from other micro operator's base stations below a certain predefined level. Unfortunately, the reduced base station transmission power will result in a degraded downlink performance of the interfering network. If the network is interference-limited (dense deployment with a high network load) the impact will be small. However, the performance of a noise-limited network (sparse deployment and/or low network load) will be considerably reduced, as demonstrated by the curves in Fig. 3 and discussed in more detail in [9]. In this study, the assumption is that for the imbalance scenario ( $\mathrm{uO} 1$ 
TABLE I. ASSUMED PARAMETER VALUES

\begin{tabular}{|c|c|}
\hline Parameter & Value \\
\hline Center frequency & $3.6 \mathrm{GHz}$ \\
\hline Total number of RBs & 100 \\
\hline Bandwidth of a RB & $180 \mathrm{kHz}$ \\
\hline $\begin{array}{l}\text { BS minimum and maximum } \\
\text { transmission power }\end{array}$ & $0 \mathrm{dBm}(\min ), 24 \mathrm{dBm}(\max )$ \\
\hline $\begin{array}{l}\text { MT minimum and maximum } \\
\text { transmission power }\end{array}$ & $-40 \mathrm{dBm}(\min ), 23 \mathrm{dBm}(\max )$ \\
\hline Receiver noise figure & $12 \mathrm{~dB}(\mathrm{BS}), 9 \mathrm{~dB}(\mathrm{MT})$ \\
\hline BS antenna gain & $5 \mathrm{dBi}$ (omnidirectional) \\
\hline MT antenna gain & $0 \mathrm{dBi}$ (omnidirectional) \\
\hline $\begin{array}{l}\text { Maximum spectral efficiency } \\
\text { (DL: } 64 \text { QAM, } 2 \text { streams) } \\
\text { (UL: } 64 \text { QAM, } 1 \text { stream) }\end{array}$ & $\begin{array}{l}10.6 \mathrm{bbs} / \mathrm{Hz}(\mathrm{DL}) \\
5.3 \mathrm{bps} / \mathrm{Hz}(\mathrm{UL})\end{array}$ \\
\hline Uplink power control target & $\begin{array}{l}-75 \mathrm{dBm}(1 \mathrm{BS}, 1 \mathrm{user}) \\
-70 \mathrm{dBm}(12 \mathrm{BS}, 8 \%, 50 \% \text { load }) \\
-65 \mathrm{dBm}(12 \mathrm{BS}, 90 \% \text { load })\end{array}$ \\
\hline Path loss compensation factor & 0.8 \\
\hline
\end{tabular}

has deployed one base station and is serving only one user), only $\mathrm{uO} 2$ is adjusting the base station transmission power based on the measurements performed by $\mathrm{uO} 1$. However, for the load imbalance (uO1 has deployed 12 base stations and the average network load is either $8 \%$ or $50 \%$ ) and balanced ( $\mathrm{uO} 1$ has deployed 12 base stations and the average network load is equal to $90 \%$ ) scenarios, both micro operators are adjusting the base station transmission powers, and the same interference threshold $\left(I_{t h}\right)$ is assumed for both.

Results for the load imbalance $(8 \%)$ scenario are presented in Fig. 4. Three different sets of curves are shown for uO1: average uplink throughput loss and average downlink throughput loss compared to a single operator deployment, and average downlink throughput loss compared to a reference deployment (i.e., a single operator deployment without any reduction of the base station transmission power). Furthermore, three different values of $I_{t h}$ have been assumed: $-70 \mathrm{dBm},-75 \mathrm{dBm}$ and $-80 \mathrm{dBm}$.

As can be noticed, a lower $I_{t h}$, i.e., a lower limit for the interfering base station transmission power, will result in a considerably reduced uplink throughput loss compared to the single operator deployment. The reason for this is that the main cause for the uplink performance loss is in fact the DL-to-UL interference from the base stations belonging to the other operator and not the ULto-UL interference from the co-existing mobile terminals [10]. Also in downlink the level of the inter-operator interference is reduced as a result of the lower base station transmission powers for the interfering network. However, at the same time the level of the received carrier power is reduced as well. Since both networks are reducing the base station powers in the same way, the downlink signal-to-interference ratio will stay the same,

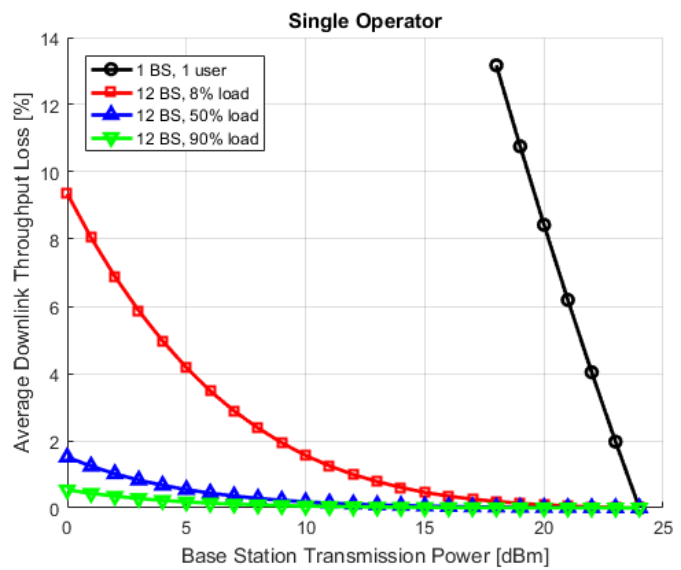

Fig. 3. Average downlink throughput loss for the single operator scenario as a function of the base station transmission power.

but due to the receiver noise power, the SINR becomes worse together with the reduced $I_{t h}$. When the buildings are close-by, the impact of the interference control on the base station transmission power becomes large, having also a noticeable impact on the SINR, in particular for the noise-limited users located deeper inside the building. But since the SINR values still quite often exceed the threshold for the maximum achievable bit rate, the impact on the actual user throughput becomes small. When the buildings are located farther away, the level of the worst case inter-operator interference is low enough to allow the use of higher base station powers. Therefore, only a marginal impact on the average user performance can be noticed. However, the downlink throughput losses compared to the reference deployment are much more visible, which is a direct result of the reduced downlink coverage in case of a noise-limited network.

Results for the required MSD between the $5 \mathrm{G}$ indoor micro operator deployments as a function of the $I_{t h}$ are presented in Fig. 5. Here, the required MSD is defined as the maximum of the required downlink MSD and the required uplink MSD. Curves are shown for four different deployment scenarios and for three different levels of the maximum allowed average performance loss for $\mathrm{uO} 1$. The results demonstrate how the required MSD can be considerably reduced by controlling the base station transmission power based on the measurements performed by the victim network. In fact, if a performance loss up to $5 \%$ is allowed, the required MSD can be reduced to zero for all the evaluated deployment scenarios. However, in case of the more stringent performance loss requirements, the deployments with a higher degree of imbalance between the micro operators will still experience non-zero MSDs even with the applied interference control mechanism. For example, if a performance loss of up to $1 \%$ is allowed, the required MSD can be reduced from $640 \mathrm{~m}$ to $270 \mathrm{~m}$ (imbalance), $370 \mathrm{~m}$ to $190 \mathrm{~m}$ (load imbalance $(8 \%)$ ), $420 \mathrm{~m}$ to $30 \mathrm{~m}$ (load imbalance $(50 \%)$ ) and $310 \mathrm{~m}$ to $0 \mathrm{~m}$ (balanced scenario). 

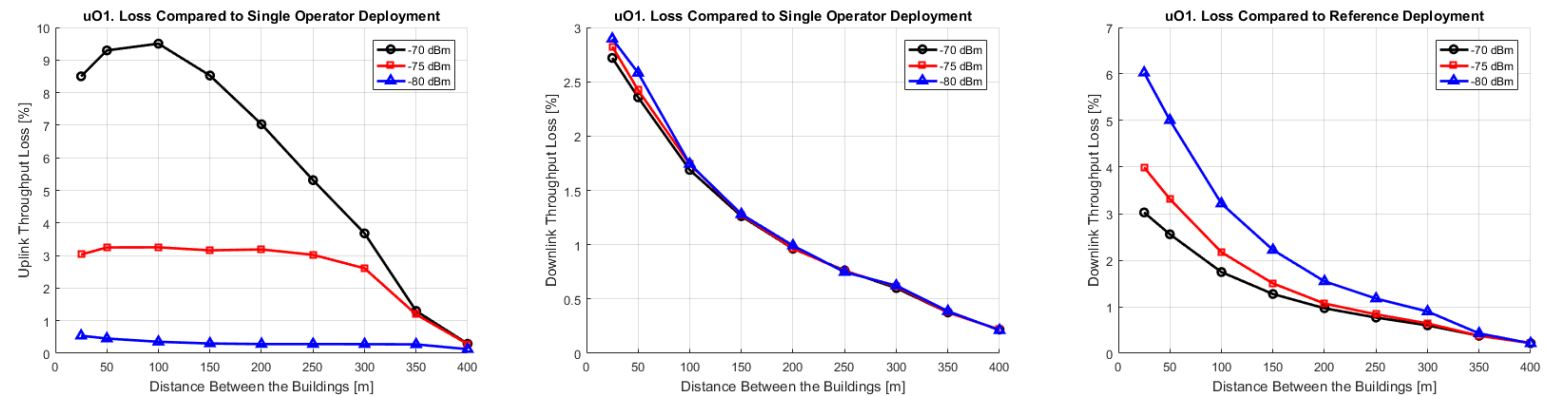

Fig. 4. Average uplink and downlink throughput reduction as a function of the distance between the buildings, assuming three different values for the interference threshold. uO1 network is assumed to consist of 12 base stations, and the network is assumed to be serving one user.
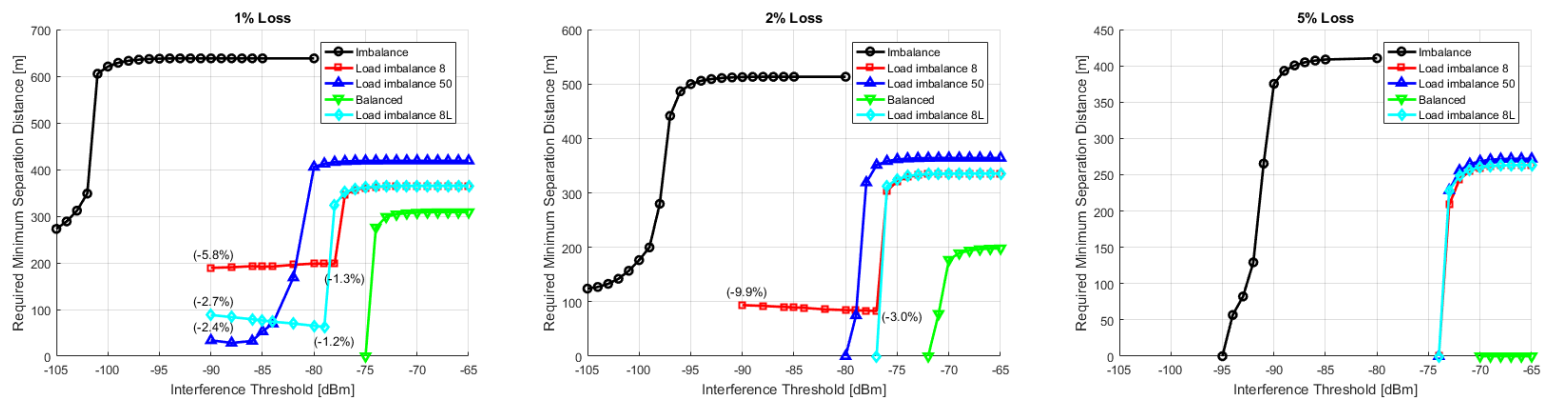

Fig. 5. Required minimum separation distance between the buildings as a function of the interference threshold for different deployment scenarios and maximum allowed performance losses. The values inside parenthesis indicate the average downlink throughput losses compared to the reference deployment.

For some of the deployment scenarios, the gain of applying a lower $I_{t h}$ becomes small after a certain point, or there may even be a loss in terms of the required MSD. This is due to the fact that for the lower $I_{t h}$ values the MSD has become limited by the downlink performance, where the gains achieved by lowering the base station transmission power even further are only marginal, or even negative. The cost of applying a lower $I_{t h}$ is confirmed also by the corresponding downlink performance losses compared to the reference deployment, as shown by the values inside parenthesis. Thus, even though the loss compared to the single operator deployment satisfies the requirement of $1 \%$ or $2 \%$, the loss compared to the reference deployment may at the same time be as large as $10 \%$.

It becomes also clear that the required $I_{t h}$ resulting in the smallest MSD is highly scenario-dependent. It is quite straightforward to understand that the performance of a noise-limited deployment is quite sensitive to external interference [10] (suggesting a lower $I_{t h}$ for the interfering network) and reduction of the serving base station transmission power (calling for a higher $I_{t h}$ for the victim network). At the same time, the situation is different for an interference-limited deployment: A dense victim network with a high load tolerates a higher level of external interference [10], allowing a higher level of $I_{t h}$ to be applied for the interfering network. Similarly, a dense interfering network with a high load can apply a lower level of $I_{t h}$ without any remarkable impact on the downlink performance. The downside for this kind of scenario-based approach is that both the $I_{t h}$ and the base station transmission power would not be static, i.e., depending only on the network layout (i.e., on the coupling losses between the mobile terminals and the serving base stations), but they would have to be adjusted together with the load of the neighboring micro operator networks.

An alternative approach would be to keep the same static $I_{t h}$ values as before, but to consider the load of the interfering network when estimating the level of the received inter-operator interference. Thus, to assume the actual base station activities $\left(\tau_{k}\right)$ in (9) instead of assuming that all base stations are active. With the modified approach, a high-loaded network would be more likely to limit the base station transmission powers compared to the low-loaded network, even when the same $I_{t h}$ is applied for both. Performance of the modified interference control mechanism has been evaluated for the load imbalance $(8 \%)$ scenario, and the results are shown in Fig. 5 (see the curve labeled as Load imbalance $8 L$ ). As can be noticed, the required MSD does indeed become considerably smaller, allowing the MSD to be equal to zero even when the requirement for the maximum performance loss is set to $2 \%$.

\section{CONCLUSIONS}

Local small cell deployments by different stakeholders complementing the coverage of the MNOs' outdoor networks have gained increasing attention in the development of the future $5 \mathrm{G}$ networks. Local high- 
quality wireless networks are particularly interesting for vertical sectors' high-traffic areas and deployments in the higher frequency bands. This requires new business and spectrum sharing models that allow different stakeholders to become $5 \mathrm{G}$ micro operators and to obtain local spectrum micro licenses. Furthermore, interference between the different micro operators becomes a critical aspect to consider, in particular when the operators are assumed to be sharing the same channel. There, the regulators need to define the required minimum separation distances between the neighboring micro operators so that the level of the received inter-operator interference is sufficiently low, while not restricting the efficiency of the spectrum usage.

The performance of a simple interference control mechanism between two uncoordinated micro operators utilizing dynamic TDD in the $3.6 \mathrm{GHz}$ band has been evaluated in this paper. In the evaluated mechanism, the victim micro operator estimates the worst case downlink interference received from the interfering micro operator. Then, the measurement is compared to a pre-defined interference threshold to define the appropriate scaling factor for the base station transmission power to be applied within the interfering micro operator's network. The obtained system simulation results indicate that the required minimum separation distance between the operators can be considerably reduced with the help of the introduced interference control mechanism. However, the results also show that the most appropriate interference threshold is highly scenario-dependent: it will depend both on the layout and the load of the neighboring micro operator networks.

A topic for future research is to enhance the interference control model so that the interference threshold would become self-adjusting, depending on the actual network layouts and the load situation. Furthermore, the performance analysis should be extended to cover the impact of multiple micro operators as well as deployment scenarios within higher frequency bands, taking into account the impact of beamforming.

\section{ACKNOWLEDGMENT}

This research in the "Micro-operator concept for boosting local service delivery in 5G (uO5G)" project has been financially supported by Business Finland and by Academy of Finland 6Genesis Flagship (grant 318927).

\section{REFERENCES}

[1] J. Zander, "Beyond the ultra-dense barrier: Paradigm shifts on the road beyond 1000x wireless capacity", IEEE Wireless Communications, vol. 24, no. 3, pp. 96-102, Jan. 2017.

[2] M. D. P. Guirao, A. Wilzeck, A. Schmidt, K. Septinus and C. Thein, "Locally and temporary shared spectrum as opportunity for vertical sectors in 5G," IEEE Network, vol. 31, no. 6, pp. 24-31, Nov./Dec. 2017.
[3] ETSI, "Feasibility study on temporary spectrum ascess for local high-quality wireless networks," ETSI TR 103588. Feb. 2018.

[4] M. Matinmikko, M. Latva-aho, P. Ahokangas, S. Yrjölä, and T. Koivumäki, "Micro operators to boost local service delivery in 5G", Wireless Personal Communications, vol. 95, no. 1, pp. 69-82, Jul. 2017.

[5] M. Matinmikko, A. Roivainen, M. Latva-aho, and K. Hiltunen, "Interference study of micro licensing for $5 \mathrm{G}$ micro operator small cell deployments", in Proc. Conference on Cognitive Radio Oriented Wireless Networks and Communications (CrownCom), Lisboa, Portugal, Sep. 2017.

[6] M. Matinmikko, M. Latva-aho, P. Ahokangas, and V. Seppänen, "On regulations for 5G: Micro licensing for locally operated networks", Telecommunications Policy, in press.

[7] P. Ahokangas, M. Matinmikko, S. Yrjölä and I. Atkova, "Disruptive revenue models for future micro operator driven mobile business ecosystem", in Proc. The 24th Nordic Academy of Management Conference (NFF), Bodo, Norway, Aug. 2017.

[8] S. Parkvall, E. Dahlman, A. Furuskär and M. Frenne, "NR: The new 5G radio access technology", IEEE Communications Standards Magazine, vol. 1, no. 4, pp. 24-30, Dec. 2017.

[9] K. Hiltunen, M. Matinmikko-Blue, M. Latva-aho, "Impact of interference between neighboring $5 \mathrm{G}$ micro operators", Wireless Personal Communications, vol. 100, no.1, pp. 127-144, May 2018.

[10] K. Hiltunen, M. Matinmikko-Blue, "Performance of neighboring indoor $5 \mathrm{G}$ micro operators with dynamic TDD", in Proc. European Conference on Networks and Communications (EuCNC) 2018, Ljubljana, Slovenia, Jun. 2018.

[11] K. Hiltunen, M. Matinmikko-Blue, "Propagation model for evaluating the interference between neighboring indoor micro operators", in Proc. IEEE Vehicular Technology Conference (VTC) 2018 Spring, Porto, Portugal, Jun. 2018.

[12] 3GPP, "Study on channel model for frequencies from 0.5 GHz to $100 \mathrm{GHz}$ ", 3GPP TR 38.901, V14.1.1, Jul. 2017.

[13] E. Damasso, and L. M. Correia (ed.), “COST 231 Final Report: Digital mobile radio towards future generation systems", Tech. Rep., 1999.

[14] E. Semaan, F. Harrysson, A. Furuskär, and H. Asplund, "Outdoor-to-indoor coverage in high frequency bands", in Proc. IEEE Globecom 2014 Workshop - Mobile Communications in Higher Frequency Bands, Austin, TX, USA, Dec. 2014.

[15] J.-E. Berg, "Building penetration loss along urban street microcells", in Proc. IEEE International Symposium on Personal, Indoor and Mobile Radio Communications (PIMRC) 1996, Taipei, Taiwan, Oct. 1996.

[16] J.-E. Berg, "A recursive method for street microcell path loss calculations", in Proc. IEEE International Symposium on Personal, Indoor and Mobile Radio Communications (PIMRC) 1995, Toronto, Canada, Sep. 1995. 